\title{
Porphyries hépatiques et chirurgie buccale. A propos d'un cas
}

\section{Hepatic porphyrias and oral surgery. Case report}

\author{
LAURENCE NOEL ${ }^{*}$, VINCENT FURON*, BENOIT LEFEVRE*, CEDRIC MAUPRIVEZ*.
}

\section{RÉSUMÉ}

Les porphyries sont des anomalies, héréditaires ou acquises, touchant certaines enzymes spécifiques de la voie de la biosynthèse de l'hème. Ces pathologies sont classées en deux groupes, hépatiques ou érythropoïétiques, en fonction du tissu dans lequel prédomine le trouble métabolique. Le cas présenté rapporte la prise en charge en chirurgie buccale d'une patiente atteinte d'une porphyrie aiguë intermittente. L'existence ou la persistance d'un foyer infectieux et/ou inflammatoire, l'utilisation d'anesthésiques locaux de type amide, la prescription de médicaments dits "porphyrinogéniques " sont des facteurs susceptibles de déclencher une crise aiguë potentiellement grave. (Med Buccale Chir Buccale 2005; 11: 103-110).

mots clés: porphyries hépatiques, odontologie, chirurgie buccale, médicaments, patient à risque

médecine

buccale chirurgie buccale

VOL. $11, \mathrm{~N}^{\circ} 2$ 2005

page 103

\section{SUMMARY}

Porphyrias are a group of hereditary or acquired abnormalities characterized by defective enzymes in the heme biosynthetic pathway. Porphyrias are classified as either hepatic or erythropoietic depending on the primary organ in which metabolic disorder takes place. This case report presents the dental management of a female patient suffering from acute intermittent porphyria. Acute attacks, sometimes lethal, are often induced by precipitating factors such as infection, drugs and local anesthetics derived from amide group. (Med Buccale Chir Buccale 2005; 11:103-110).

key words: hepatic porphyrias, dental management, oral surgery, drugs 
médecine buccale chirurgie buccale

VOL. $11, \mathrm{~N}^{\circ} 2$ 2005

page 104
Les porphyries constituent un groupe de pathologies se caractérisant par une accumulation tissulaire de porphyrines et une élimination accrue urinaire et fécale de porphyrines ou de leurs précurseurs, principalement l'acide delta-aminolévulinique (ALA) et le porphobilinogène (PBG). Les porphyrines sont des pigments rouges formés de 4 noyaux pyrroliques. La liaison avec un atome ferreux $(\mathrm{Fe} 2+)$ au centre de ce groupement constitue l'hème [1]. Environ $85 \%$ de l'hème produit dans l'organisme est synthétisé dans les cellules érythrocytaires pour fournir l'hème à l'hémoglobine, le reste est produit essentiellement dans le foie où l'hème est utilisé pour la synthèse des enzymes du cytochrome P450 [1-3]. Les porphyries ont pour origine un déficit en une des enzymes intervenants dans la biosynthèse de l'hème [1-17] (Fig 1). Les porphyries peuvent être héréditaires ou acquises. Les premières sont les plus fréquentes et pour la plupart de transmission autosomique dominante. Elles sont classées en deux grands groupes: les porphyries hépatiques et les porphyries érythrocytaires, en fonction du tissu dans lequel prédomine le trouble métabolique. Les porphyries hépatiques représentent plus de $90 \%$ de l'ensemble des porphyries [5]. Leur fréquence est faible, le nombre de sujets atteints d'une porphyrie en France était estimé à environ 50.000 en 2004 [6]. Elles surviennent généralement chez la femme (sex-ratio H/F de 1/5) entre 15 et 45 ans [1,6-10]. Les porphyries hépatiques regroupent les porphyries hépatiques aiguës et la porphyrie cutanée tardive $[1,3,6,8,9,11]$. Les porphyries hépatiques aiguës (PHA) comprennent: la porphyrie aiguë intermittente secondaire à un déficit en porphobilinogène désaminase, la porphyrie variegata secondaire à un déficit en proto-oxydase et la coproporphyrie héréditaire secondaire à un déficit en copro-oxydase. Les PHA se caractérisent par la survenue de crise(s) aiguë(s) associant principalement un syndrome abdominal douloureux et des troubles neuropsychiatriques $[1,3,6,8-10]$. Ces derniers (paralysies, insuffisance respiratoire et troubles psychiatriques) font la gravité de ce type de porphyrie. La quatrième porphyrie hépatique, la porphyrie cutanée tardive, est due à un déficit en uro-décarboxylase et ne comporte, comme son nom l'indique, que des lésions cutanées [6,8,11,12]. Les porphyries érythropoïétiques comprennent la porphyrie érythropoïétique congénitale (ou maladie de Günther) et la protoporphyrie érythropoïétique. Elles sont respectivement dues à un déficit en uro-synthétase et en ferrochélatase. Elles sont en général diagnostiquées dans la petite enfance devant la présence d'urines rouges, d'une splénomégalie et d'une anémie hémolytique. Les porphyrines s'accumulent principalement dans la peau, les dents et les os. De ce fait, la peau présente une hyperpigmentation localisée sur les zones exposées au soleil (mains, face), les dents ont une couleur brun rougeâtre, et des lacunes osseuses disséminées dans les maxillaires sont fréquemment observées [2,4].

A l'exception des PHA, qui peuvent s'accompagner de crise aiguë, la porphyrie cutanée tardive et les porphyries érythropoïétiques ne nécessitent pas de précautions spécifiques lors de soins dentaires ou d'une intervention de chirurgie buccale [1,9,13].

Le cas rapporté concerne la prise en charge en odonto-stomatologie d'une patiente atteinte d'une porphyrie aiguë intermittente (PAI).

\section{OBSERVATION}

Une femme, âgée de 46 ans, est adressée par le service de néphrologie et d'hémodialyse pour des douleurs chroniques d'origine dentaire.

L'interrogatoire médical révèle que cette patiente souffre d'une PAI, diagnostiquée à l'âge de 20 ans au cours d'un épisode neurologique sévère. Plusieurs crises aiguës sont retrouvées à l'anamnèse, la plus récente datant de novembre 2003. La patiente est en hémodialyse depuis septembre 2002 et en attente d'une transplantation rénale. Dans les antécédents, on note également un carcinome adénoïde kystique de la lèvre supérieure (T2NoMo) traité par chirurgie et radiothérapie externe et une hypertension artérielle instable. Son traitement médical actuel comporte un traitement antihypertenseur associant un inhibiteur calcique (amlodipine) et un béta-bloquant (aténolol), un anti-ischémique (buflomédil), un anti-ulcéreux (oméprazole), du carbonate de calcium et des résines hypokaliémiantes pour la 
prophylaxie des complications hydro-électrolytiques de son insuffisance rénale chronique.

L'examen clinique exobuccal objective une hyperpigmentation de la face (Fig.2) et des avant-bras, ainsi qu'une amyotrophie invalidante touchant les mains (Fig. 3) et les pieds. Un état d'anxiété marqué est également observé. L'examen clinique endobuccal révèle une arcade maxillaire totalement édentée et la présence 5 dents (33, 34, 35, 43 et 44) sur l'arcade mandibulaire. II existe une fistule en regard de l'apex de la 43. Un examen radiologique, associant un cliché panoramique (Fig. 4) et des radiographies rétro-alvéolaires, montre une image radiotransparente circonscrite à l'apex de la 43. Devant une hygiène bucco-dentaire insuffisante et pour raisons prothétiques, il est difficile d'envisager un traitement conservateur pour les dents restantes.

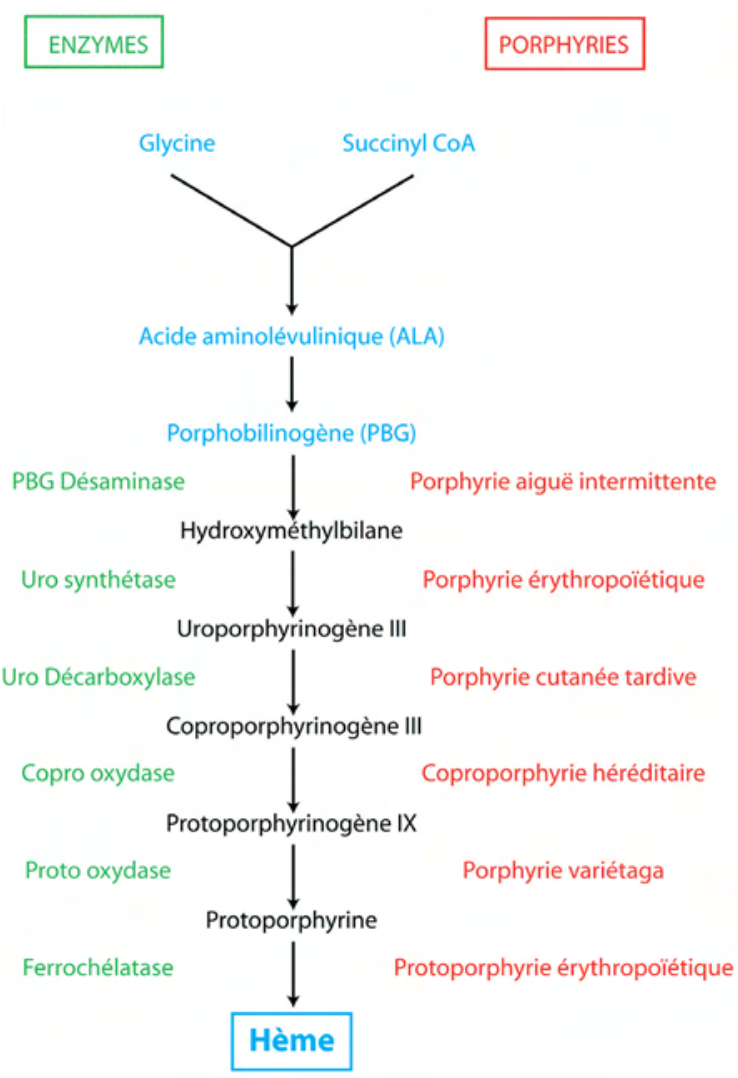

Figure 1: Déficits enzymatiques spécifiques et porphyries.

Porphyrias and their specific defective enzymes.
L'intervention chirurgicale est programmée sous surveillance médicale. Les extractions dentaires de 33, 34, 35, 43 et 44 sont réalisées sous anesthésie locale par infiltration sous muqueuse de procaïne adrénalinée à 1/200 000 (protocole défini par le Centre français des Porphyries) [4,6]. L'hémostase chirurgicale est obtenue par une suture hermétique (Vicryl 3/0®). L'analgésie post-opératoire repose sur la prescription d'un anti-inflammatoire non stéroïdien à doses antalgiques (kétoprofène $25 \mathrm{mg}$ ). Des antiseptiques locaux à base de chlorhexidine sous forme de bains de bouche sont également prescrits. Un contrôle de la cicatrisation muqueuse et la dépose des points de suture sont effectués au $7^{\mathrm{e}}$ jour post-opératoire. La réalisation d'une prothèse amovible complète est différée à 3 mois.

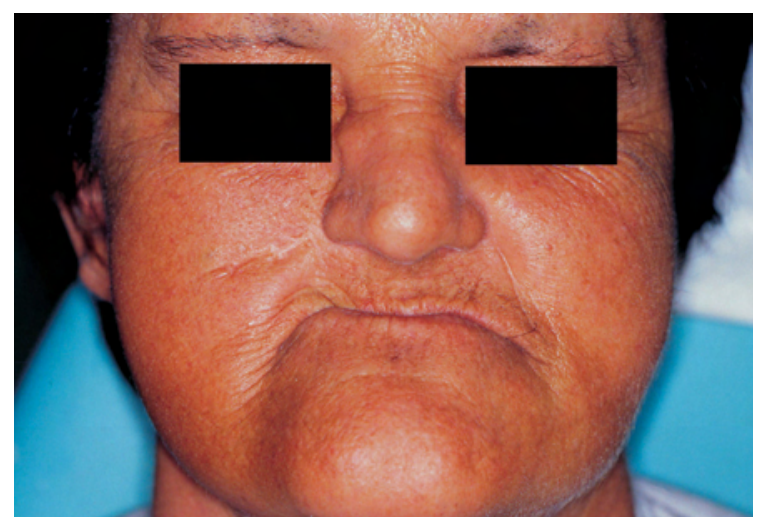

médecine

buccale chirurgie buccale

VOL. $11, \mathrm{~N}^{\circ} 2$ 2005

page 105
Figure 2 : Vue exobuccale de la patiente. Hyperpigmentation de la face.

Exobuccal view of the patient. Hyperpigmentation of the face.

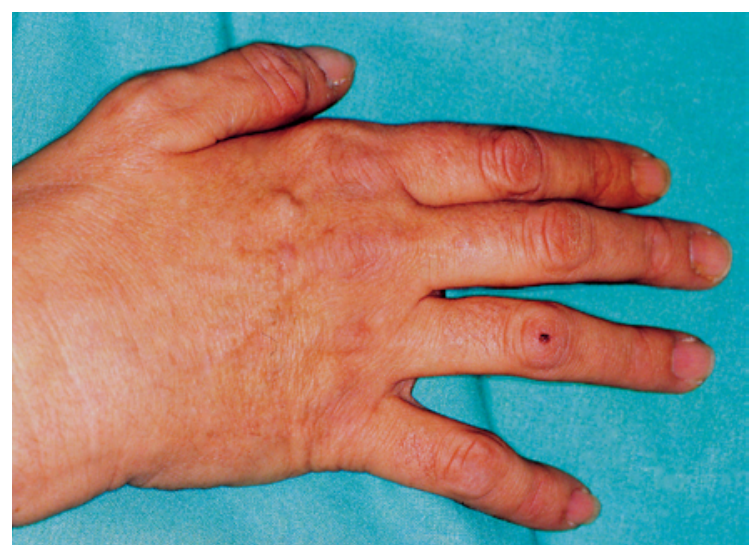

Figure 3 : Vue d'une main de la patiente montrant l'amyotrophie.

View of a hand of the patient showing the muscular damage. 


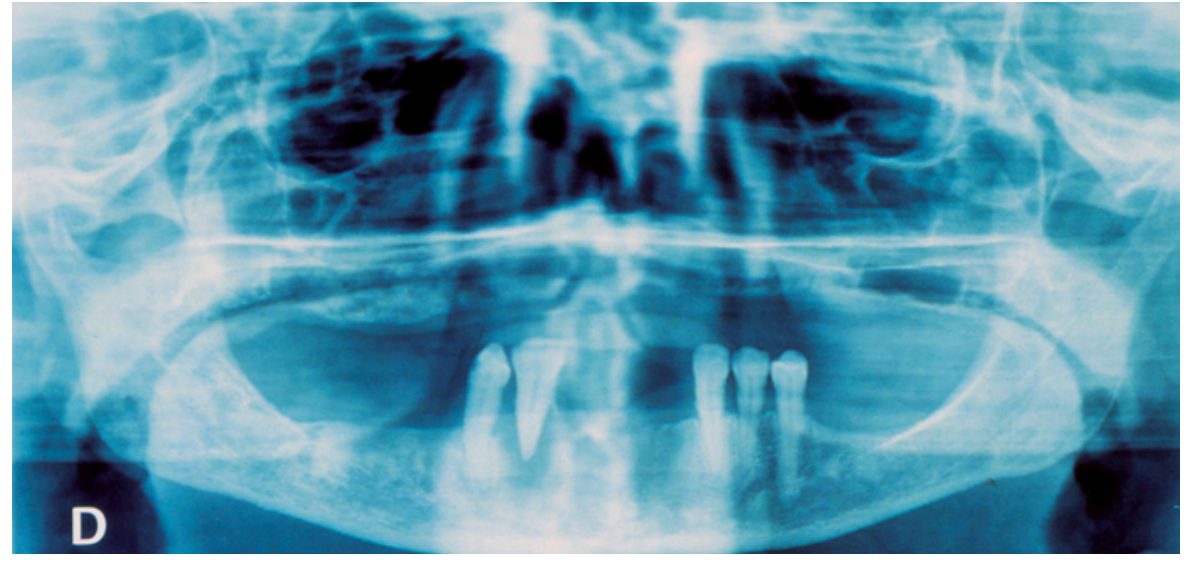

Figure 4 : Examen radiographique : cliché panoramique. Radiographic examination: orthopantomograph. médecine

buccale

chirurgie

buccale

VOL. $11, \mathrm{~N}^{\circ} 2$ 2005

page 106

\section{COMMENTAIRES}

La gravité d'une PHA repose sur le risque de survenue d'une crise aiguë de porphyrie avec ses complications neurologiques. La symptomatologie d'une crise aiguë de porphyrie hépatique (CAPH) est identique pour les trois PHA (la PAI, la porphyrie variegata et la coproporphyrie héréditaire). Le tableau clinique typique est dominé par un syndrome abdominal aigu (95\% des cas) [5] et des troubles neuropsychiatriques (90\% des cas) $[3,5,8,9]$. Les douleurs abdominales sont intenses, sans localisation prédominante, volontiers irradiantes vers la région lombaire et/ou les cuisses. S'y ajoutent une constipation, des nausées, des vomissements à répétition dont l'abondance peut entraîner des troubles hydroélectrolytiques. Les troubles psychiatriques sont le plus souvent mineurs, à type d'anxiété, d'apathie, d'insomnie, voire d'un état dépressif. Plus rarement sont rapportées des bouffées délirantes, une hystérie ou une confusion mentale [1,6]. Des manifestations convulsives sont retrouvées dans environ $20 \%$ des cas [5]. Enfin, la crise aiguë peut s'accompagner dans $2 / 3$ des cas, d'une hypertension artérielle et/ou d'une tachycardie. La découverte d'urines «rouge porto » est un élément majeur du diagnostic [3,10]. Cependant, il est rare que cette coloration soit présente à la miction, cette coloration apparaissant en général après quelques heures d'exposition à la lumière. Les complications d'une CAPH sont dominées par les séquelles neurologiques et l'insuffisance rénale chronique [3,5,11,14,15]. L'atteinte neurologique se manifeste par une neuropathie périphé- rique avec principalement une atteinte des neurones moteurs [2,8]. Celle-ci affecte d'abord les muscles proximaux. Progressivement s'installe une amyotrophie généralisée. Son évolution peut conduire à une paralysie des muscles respiratoires et être à l'origine d'une détresse respiratoire progressive et sévère pouvant mener au décès du patient.

Des troubles sensitifs de type paresthésies sont également rencontrés.

La quasi-totalité de la symptomatologie de la CAPH et de ses complications s'expliquent par l'action neurotoxique des précurseurs des porphyrines sur le système nerveux central et périphérique [5]. Les douleurs abdominales, l'hypertension artérielle et la tachycardie sont dues à l'hyperactivité sympathique. L'hyponatrémie et l'altération de la fonction rénale résultent d'une atteinte hypothalamique. Enfin les manifestations psychiatriques ainsi que les crises convulsives seraient dues à une atteinte du système nerveux central. Des taux plasmatiques et urinaires très élevés d'ALA et de PBG sont effectivement observés pendant les crises. De fait, le dosage urinaire de l'ALA et du PBG permet de confirmer le diagnostic de CAPH (taux de l'ALA multiplié par 10 ; celui du PBG par 50) [4]. Inversement, des taux normaux d'ALA et de PBG excluent formellement une PHA comme cause des symptômes observés. A distance de la crise, il importe de préciser le type de PHA dont le patient est atteint, par le dosage de l'enzyme déficitaire spécifique de la porphyrie $[8,16]$. Un déficit en porphobilinogène désaminase identifie une PAI. 
Le traitement d'une CAPH repose actuellement sur l'administration par voie intraveineuse d'un analogue de l'hème, l'arginate d'hémine (Normosang $®)$ [ $[3,8,6,10]$. L'hème qui agit par rétrocontrôle en inhibant la synthèse de l'ALA-synthétase, bloque ainsi la formation de l'ALA et du PBG $[3,5,8]$. La posologie recommandée est de 3 à $4 \mathrm{mg} \cdot \mathrm{kg}^{-1} \cdot \mathrm{j}^{-1}$ dilués dans $200 \mathrm{ml}$ de sérum physiologique, perfusés en 30 minutes, pendant 4 jours [2,9,5]. L'efficacité clinique et biologique est bonne. Ce traitement spécifique permet, en 48 à 72 heures, de faire disparaître le syndrome abdominal, les signes cardiovasculaires et de normaliser l'excrétion urinaire de l'ALA et du PBG. Par contre, l'effet sur les complications neurologiques (neuropathie périphérique, paralysie) est faible ou nul [2]. Des perfusions de glucose à hautes doses (400 à 500 g. $\left.24 \mathrm{~h}^{-1}\right)$ sont également préconisées mais leur efficacité clinique est inconstante $[1,5,8,15]$. Un traitement symptomatique antalgique et sédatif est impératif. II associe toujours péthidine (Péthidine $\AA$ ) et chlorpromazine (Largactil $囚$ ). Malgré les progrès thérapeutiques, le pronostic vital et neurologique de la PHA reste sévère $[10,13,14]$. Le traitement doit donc, avant tout, être préventif car, dans $50 \%$ des cas, la CAPH est déclenchée par un facteur favorisant connu [6]. II est également rapporté une plus forte prévalence d'hépatocarcinome chez les patients atteints de porphyrie hépatique [16].

Dans la pratique odonto-stomatologique, les patients atteints de PHA ne présentent aucun risque infectieux ou hémorragique particulier. Le risque majeur encouru est le déclenchement possible d'une crise aiguë consécutive aux soins dentaires. Les principaux facteurs susceptibles de déclencher une CAPH sont nombreux: l'infection, le stress, le jeûne, la consommation de certains médicaments et l'injection d'anesthésiques locaux de type amide [4,5,7-9,13]. En conséquence, tout état infectieux et/ou inflammatoire, aigu ou chronique, doit faire l'objet d'un traitement curatif rigoureux. Toute agression psychique doit être évitée. En ce qui concerne la prescription, une liste non exhaustive des médicaments autorisés et proscrits est proposée dans les Tab. 1 et 2 . En effet, les médicaments dont le métabolisme hépatique implique le cytochrome P450 et tous les xénobiotiques inducteurs enzymatiques (barbituriques, sulfamides, érythromycine, carbamazépine, alcool...) augmentent les besoins en hème. II en résulte une augmentation de l'activité de la ALA-synthétase avec pour conséquence chez les patients atteints d'une porphyrie, du fait de leur déficit enzymatique, une augmentation de la synthèse de l'ALA et du PBG [9] (Fig 5).

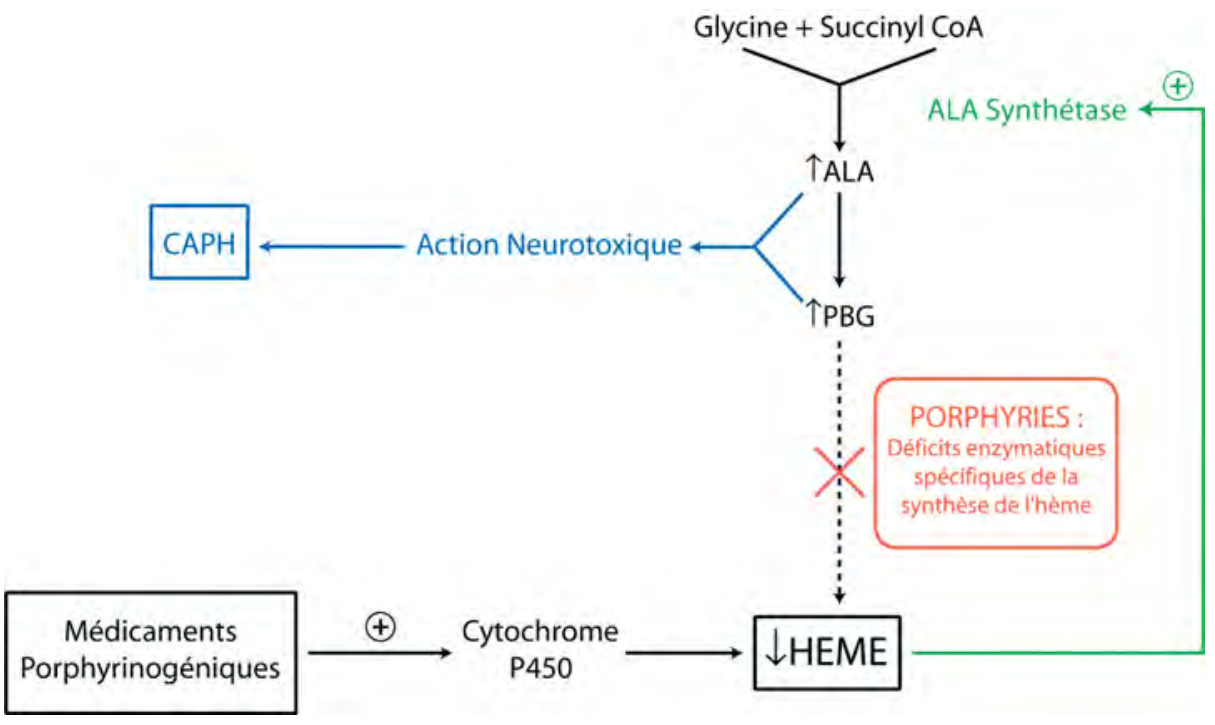

Figure 5 : Schéma d'accumulation des précurseurs ALA et PBG sous l'action des médicaments porphyrinogéniques chez un sujet atteint de porphyrie (D'après [9]).

Accumulation of the precursors ALA and PBG following the administration of inducing drugs to patients with porphyrias. 
Tableau 1 : PORPHYRIES HEPATIQUES - MEDICAMENTS AUTORISES Liste par classe médicamenteuse (D'après [5])

Hepatic porphyrias - Drugs thought to be safe.

\begin{tabular}{|c|c|c|}
\hline $\begin{array}{l}\text { Anesthésiques locaux } \\
\text { procaiine } \\
\text { tétracaiine } \\
\text { benzocainne }\end{array}$ & $\begin{array}{l}\quad \text { Analgésiques } \\
\text { diflunisal } \\
\text { codéine } \\
\text { acide acétylsalicylique }\end{array}$ & $\begin{array}{l}\quad \text { Antibiotiques } \\
\text { acide clavulanique } \\
\text { amoxicilline } \\
\text { pénicilline } \\
\text { pivampicilline } \\
\text { clarithromycine } \\
\text { josamycine } \\
\text { pristinamycine } \\
\text { spiramycine }\end{array}$ \\
\hline $\begin{array}{l}\text { Anesthésiques généraux } \\
\text { alfentanil } \\
\text { fentanyl } \\
\text { sufentanil } \\
\text { propofol }\end{array}$ & $\begin{array}{l}\quad \text { Anti-inflammatoires } \\
\text { acide niflumique } \\
\text { acide tiaprofénique } \\
\text { kétoprofène } \\
\text { naproxène } \\
\text { rofecoxib }\end{array}$ & $\begin{array}{l}\text { Antiviraux } \\
\text { aciclovir }\end{array}$ \\
\hline $\begin{array}{l}\text { flunitrazépam } \\
\text { midazolam } \\
\text { atracurium } \\
\text { thiocolchicoside } \\
\text { protoxyde d'azote }\end{array}$ & glucocorticoïdes & $\begin{array}{l}\text { Antifongiques } \\
\text { amphotéricine B }\end{array}$ \\
\hline
\end{tabular}

Tableau 2 : PORPHYRIES HEPATIQUES - MEDICAMENTS INTERDITS

Liste par classe médicamenteuse (D'après [5])

Hepatic porphyrias - Drugs to avoid.

\begin{tabular}{|c|c|c|}
\hline $\begin{array}{l}\text { Anesthésiques locaux } \\
\text { articaïne } \\
\text { lidocaïne } \\
\text { mépivacaïne } \\
\text { prilocaïne } \\
\text { ropivacaïne }\end{array}$ & $\begin{array}{l}\text { Analgésiques } \\
\text { paracétamol } \\
\text { propoxyphène } \\
\text { floctafénine } \\
\text { tramadol }\end{array}$ & $\begin{array}{l}\text { Antibiotiques } \\
\text { clindamycine } \\
\text { métronidazole } \\
\text { ornidazole } \\
\text { tinidazole } \\
\text { roxithromycine }\end{array}$ \\
\hline $\begin{array}{l}\text { Benzodiazépines } \\
\text { Anti-histaminiques } \\
\text { diazépam } \\
\text { hydroxyzine }\end{array}$ & $\begin{array}{l}\text { Anti-inflammatoires } \\
\text { acide méfénamique } \\
\text { alminoprofène } \\
\text { flurbiprofène } \\
\text { ibuprofène } \\
\text { celecoxib }\end{array}$ & $\begin{array}{l}\text { Antifongiques } \\
\text { kétoconazole } \\
\text { miconazole }\end{array}$ \\
\hline
\end{tabular}


L'effet porphyrinogénique des médicaments est rarement prédictible. Des molécules de structure très proche ou bien appartenant à la même famille pharmacothérapeutique n'ont pas forcément les mêmes effets [9,17]. Aussi, le Centre Français des Porphyries (CFP) a établi une liste de médicaments autorisés ou proscrits.

Elle est disponible à l'adresse suivante: Service de Biochimie, Pr DEYBACH, Hôpital Louis Mourier, 178 rue des Renouillers 92701 Colombes Cedex (Tél : 01476063 34) ou sur internet : http://www.porphyries.com.fr.

II n'existe aucune contre-indication à la réalisation d'une anesthésie locale ou générale [6]. En revanche, tous les amino-amides couramment utilisés en odonto-stomatologie (lidocaïne, mépivacaïne, prilocaïne et articaïne) sont formellement proscrits. Le seul anesthésique local autorisé est la procaïne et ses dérivés. En effet, les aminoesters sont métabolisés dans le sérum et le foie par des pseudo-cholinestérases et non par le système du cytochrome P450. La procaïne n'est disponible que sous forme d'ampoule et sans vasoconstricteur. Toute prémédication neurosédative à base d'hydroxyzine ou de diazépam doit être proscrite. Toutefois, une sédation consciente par inhalation de protoxyde d'azote est possible. Parmi les analgésiques et les antiinflammatoires non stéroïdiens, le paracétamol et l'ibuprofène sont à proscrire. Tous les glucocorticoïdes, sans exception, sont autorisés.
Concernant les antibiotiques, l'amoxicilline, la josamycine, la spiramycine et la pristinamycine peuvent être prescrits ; en revanche le métronidazole est proscrit.

Les manifestations cutanées associées aux porphyries sont dominées par une hyperpigmentation de la face et des mains [6,8,9]. La fragilité cutanée se traduit par l'apparition de vésicules ou de bulles après un traumatisme léger et l'évolution se fait lentement en laissant des cicatrices hyper- ou hypopigmentées. Fréquemment, on trouve associé une hypertrichose (région malaire, front, avant-bras) et des microkystes sur les doigts. Ces signes cutanés sont présents dans toutes les porphyries à l'exception de la PAI. Ainsi, dans le cas clinique présenté, l'hyperpigmentation ne peut donc vraisemblablement pas être attribuée à la maladie. En revanche, il existe de nombreux médicaments responsables d'une photosensibilité, notamment la chlorpromazine.

Même si les porphyries sont des maladies rares, tout odonto-stomatologiste peut être amené à prendre en charge un sujet atteint de cette affection. Le type et la sévérité de la porphyrie doivent être précisés. En dehors de la PHA, cette prise en charge ne pose pas de problème majeur. En revanche, devant une PHA, le praticien doit respecter scrupuleusement toutes les mesures préventives qui visent à éliminer les facteurs favorisants et/ou aggravants d'une CAPH.

7 - AndeRsson C, InNALA E, BACKSTROM T. Acute intermittent porphyria in women: clinical expression, use and experience of exogenous sex hormones. A populationbased study in northern Sweden. J Int Med 2003; 254 : 176-83.

8 - BADMINTON MN, EldER GH. Management of acute and cutaneous porphyrias. Int J Clin Pract 2002 ; 56 : 2728.

9 - Deybach JC, Puy H, Nordmann Y. Porphyries hépatiques et odontostomatologie. Rev Odontostomatol $1998 ; 27: 155-63$.

10 - Moore AW, Coke JM. Acute porphyric disorders. Oral Surg Oral Med Oral Pathol Oral Radiol Endod 2000 ; $90: 257-62$.

11 - MURPHY GM. Evaluation of porphyria. Photodermatol Photomed $1998 ; 14:$ 58-63. médecine

buccale

chirurgie

buccale

VOL. $11, \mathrm{~N}^{\circ} 2$ 2005

page 109
4 - GAUDY JF, ARRETo CD. Manuel d'analgésie en odontostomatologie (pp.121-66). Masson, Paris 1999.

5 - Gajdos P, NoRdmann Y. Les porphyries hépatiques (pp 444-6). In : Hématologie tome II. A. Najman, E. Verdy, G. Potron, F. Isnard. éd: Ellipses, Paris 1994.

6 - CENTRE FRANÇAIS DES PORPHYRIES. Les porphyries hépatiques aiguës. (Page consultée le 10/11/04). http ://www.porphyries.com.fr. 
12 - LIM HW. The porphyrias: an introduction. Photodermatol Photomed 1998; $14: 46-7$.

13 - Brown RS, HaYs GL, JeAnsonne MJ, LUSK SS. The management of a dental abscess in a patient with acute intermittent porphyria. Oral Surg Oral Med Oral Pathol $1992 ; 73: 575-8$.

14 - Soonawalla ZF, Orug T, Badminton mN, Elder GH, RHODES JM, BRAMHALL SR, ELIAS E. Liver transplantation as a cure for acute intermittent porphyria. Lancet 2004 ; 363: 705-6.
15 - WARHOLM C, WILCZEK H. Renal transplantation in a case of acute intermittent porphyria. J Clin Pharmacol 2003; $43: 1158-60$.

16 - PALmieri C, VIGUShIn DM, Peters TJ. Managing malignant disease in patients with porphyria. Q J Med 2004; 97: 115-26.

17 - De Verneuil h, Deybach JC, Phung N, DA Silva V, NoRDMANN Y. Study of anaesthetic agents for their ability to elicit porphyrin biosynthesis in chick embryo liver. Bioch Pharmacol 1983 ; 32 : 1011-8. médecine

buccale

chirurgie

buccale

VOL. $11, \mathrm{~N}^{\circ} 2$ 2005 